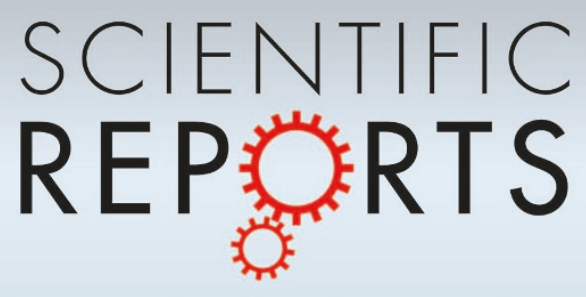

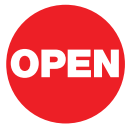

SUBJECT AREAS: NEURODEGENERATION

CELLULAR NEUROSCIENCE

MOLECULAR NEUROSCIENCE

ALZHEIMER'S DISEASE

Received

4 July 2012

Accepted

5 September 2012

Published

3 October 2012

Correspondence and requests for materials should be addressed to R.K. (rakayed@utmb.

edu)

* Current address: Neurological Research Institute, Texas

Children's Hospital, Houston, Texas.

\section{Alzheimer brain-derived tau oligomers propagate pathology from endogenous} tau

\author{
Cristian A. Lasagna-Reeves ${ }^{1,2 *}$, Diana L. Castillo-Carranza ${ }^{1,2}$, Urmi Sengupta ${ }^{1,2}$, \\ Marcos J. Guerrero-Munoz ${ }^{1,2}$, Takaki Kiritoshi' ${ }^{3}$, Volker Neugebaver ${ }^{3}$, George R. Jackson ${ }^{1,2,3}$ \\ \& Rakez Kayed ${ }^{1,2,3}$
}

${ }^{1}$ George P. and Cynthia Woods Mitchell Center for Neurodegenerative Diseases, ${ }^{2}$ Department of Neurology, ${ }^{3}$ Neuroscience and Cell Biology University of Texas Medical Branch, 301 University Blvd, Galveston, TX, 77555-1045, USA.

Intracerebral injection of brain extracts containing amyloid or tau aggregates in transgenic animals can induce cerebral amyloidosis and tau pathology. We extracted pure populations of tau oligomers directly from the cerebral cortex of Alzheimer disease (AD) brain. These oligomers are potent inhibitors of long term potentiation (LTP) in hippocampal brain slices and disrupt memory in wild type mice. We observed for the first time that these authentic brain-derived tau oligomers propagate abnormal tau conformation of endogenous murine tau after prolonged incubation. The conformation and hydrophobicity of tau oligomers play a critical role in the initiation and spread of tau pathology in the naive host in a manner reminiscent of sporadic AD.

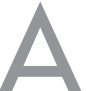

growing body of evidence suggests that protein aggregates associated with neurodegenerative diseases spread from affected to unaffected areas of the brain, indicating that prion-like transmission of these diseases contributes to the anatomic spread of pathology ${ }^{1,2}$. Extracts from $\mathrm{AD}$ brains or transgenic mouse models of $\mathrm{AD}$ can initiate amyloid plaque formation in the brain of transgenic mice over-expressing human amyloid precursor protein (APP) $)^{3,4}$. The same propagation mechanism has also demonstrated for tau. Remarkably, Goedert, Tolnay, and coworkers showed that when brain extracts obtained from transgenic mice that overexpress mutant tau (P301S) were injected into the brains of ALZ17 mice, a transgenic line overexpressing wild type human tau which normally do not form tau aggregates, tau deposition was found not only within the injection site but also in neighboring brain regions ${ }^{5}$. Moreover, two recent reports ${ }^{6,7}$, confirmed the spread of tau pathology between different brain regions, later clearly demonstrated that human tau can seed endogenous mouse tau in vivo ${ }^{7}$. This is not surprising because of the sequence homology shared between the two species ${ }^{8}$.

These observations represent major strides forward in understanding of tauopathy, but they failed to identify the tau species responsible for propagation and neurotoxicity. Moreover, the use of animal models that overexpress amyloidogenic proteins does not support the intriguing analogy to sporadic neurodegenerative diseases, since it is most likely that these seeds just accelerate amyloid deposits in transgenic mice. To date, it is still unclear whether amyloid-seeding species are able to induce amyloidosis in wild type animals, a point of great relevance in the specific case of tauopathies, where many studies have shown that endogenous murine tau aggregates in the transgenic mouse models that overexpress other aggregation-prone proteins i.e., human mutant APP, $\alpha$ synuclein, or tau ${ }^{9-11}$. Furthermore, endogenous murine tau plays a crucial role in induction of cognitive deficits in transgenic mice expressing mutant human $\mathrm{APP}^{12}$, and mediates amyloid oligomer toxicity ${ }^{13,14}$.

Recently, oligomers derived from many amyloidogenic proteins, including tau, have been implicated in neurotoxicity and neurodegeneration in amyloid diseases ${ }^{15-19}$. In spite of these compelling studies, however, the role of amyloid oligomers in disease initiation and progression in vivo remains unclear. Jucker and colleagues recently presented evidence that soluble $A \beta$ amyloid species are more effective (50-fold stronger) than larger insoluble aggregates in seeding and initiating amyloidosis in transgenic animals ${ }^{20}$. Recently, we and others have identified tau oligomers in $v^{i v 0^{19,21}}$; however, the role that these soluble oligomeric tau species play in the initiation and spread of tau pathology in vivo is largely unknown. We hypothesize that the dynamic tau oligomer ${ }^{22,23}$, which display strong hydrophobic properties similar to the prion particles described in the original work of Prusiner ${ }^{24,25}$, are responsible for tau pathology initiation and propagation. 


\section{Results}

Isolation and characterization of tau oligomers and paired helical filaments (PHF) from AD brains. To clarify the nature of authentic brain-derived tau species responsible for transmissibility and neurotoxicity, we isolated tau oligomers by immunoprecipitation (IP) techniques using the anti-tau oligomer antibody $\mathrm{T} 22^{18}$ and paired helical filaments (PHF) tau as previously described ${ }^{26}$. Tau oligomers were isolated and characterized from $3 \mathrm{AD}$ brains, no detectable tau oligomers were found in the IP from 3 age-matched control brains, this is not surprising, because as previously reported control tau oligomers are not present in control brains ${ }^{18,19}$. The seeding, LTP and in vivo experiments were performed using tau oligomers and $\mathrm{PHF}$ isolated from the same AD brain. Brain-derived tau oligomers were extensively characterized using immunoblotting, atomic force microscopy (AFM), and size-exclusion chromatography (SEC), as well as hydrophobicity and amyloid-sensitive assays with bis-1anilinonaphthalene-8-sulfonate (Bis-ANS) and thioflavin T (ThT), respectively (Fig. 1). Isolated human tau oligomers were detected by immunoblot at the $\sim 110-160 \mathrm{kDa}$ band using Tau 5, confirming that the isolated material was indeed oligomeric tau free from monomer contamination (Fig. 1a). These results correspond to a dimer/trimer as previously described ${ }^{18,19}$. The same blot was performed using control anti-IgG antibody, which failed to detect any non-specific bands. Total PBS soluble fractions from AD brains were also tested as controls (Fig. 1a). Fast protein liquid chromatography (FPLC) chromatogram of brain-derived tau oligomers detected a main peak at $\sim 150-195 \mathrm{kDa}$ (tau oligomers) (Fig. 1b). The same protocol was used for further purification of oligomers isolated by IP. We used AFM to further characterize these brain-derived oligomers (Fig. 1c) the analysis show only oligomeric tau without PHF contamination. The size distribution histogram (Fig. 1d) shows that the majority of these oligomers have a diameter of 4-8 $\mathrm{nm}$. Surprisingly, brain-derived oligomers have a higher affinity for Bis-ANS than brain-derived PHF (Fig. 1e), which implies that oligomers display more hydrophobic patches. Since recent reports implicated tau fibrils in the seeding process ${ }^{27,28}$, we isolated pure populations of brainderived PHF from the sarcosyl insoluble fractions of the same AD brains. These PHF samples were characterized by AFM (Fig. 1f), which demonstrated that they are classical PHF. As expected, the PHF exhibit strong ThT binding affinity as compared to brain-derived oligomers (Fig. 1g). The human brain-derived tau oligomers (dimer/ trimer) are SDS-stable ${ }^{19,21}$, similar to the ones detected in mouse models of tauopathy ${ }^{29,30}$.

Brain-derived tau oligomers seeding and toxicity in vitro. We investigated the ability of brain-derived tau oligomers to seed and exert toxicity in vitro. A body of literature has emerged over the last two decades documenting the ability of amyloid and tau fibrils to seed and propagate aggregation; this process is well standardized and documented $^{31-36}$. Lately, it has been shown that amyloid oligomers also can seed and propagate in vitro ${ }^{37,38}$. Recently, we discovered that tau oligomers prepared from recombinant tau can
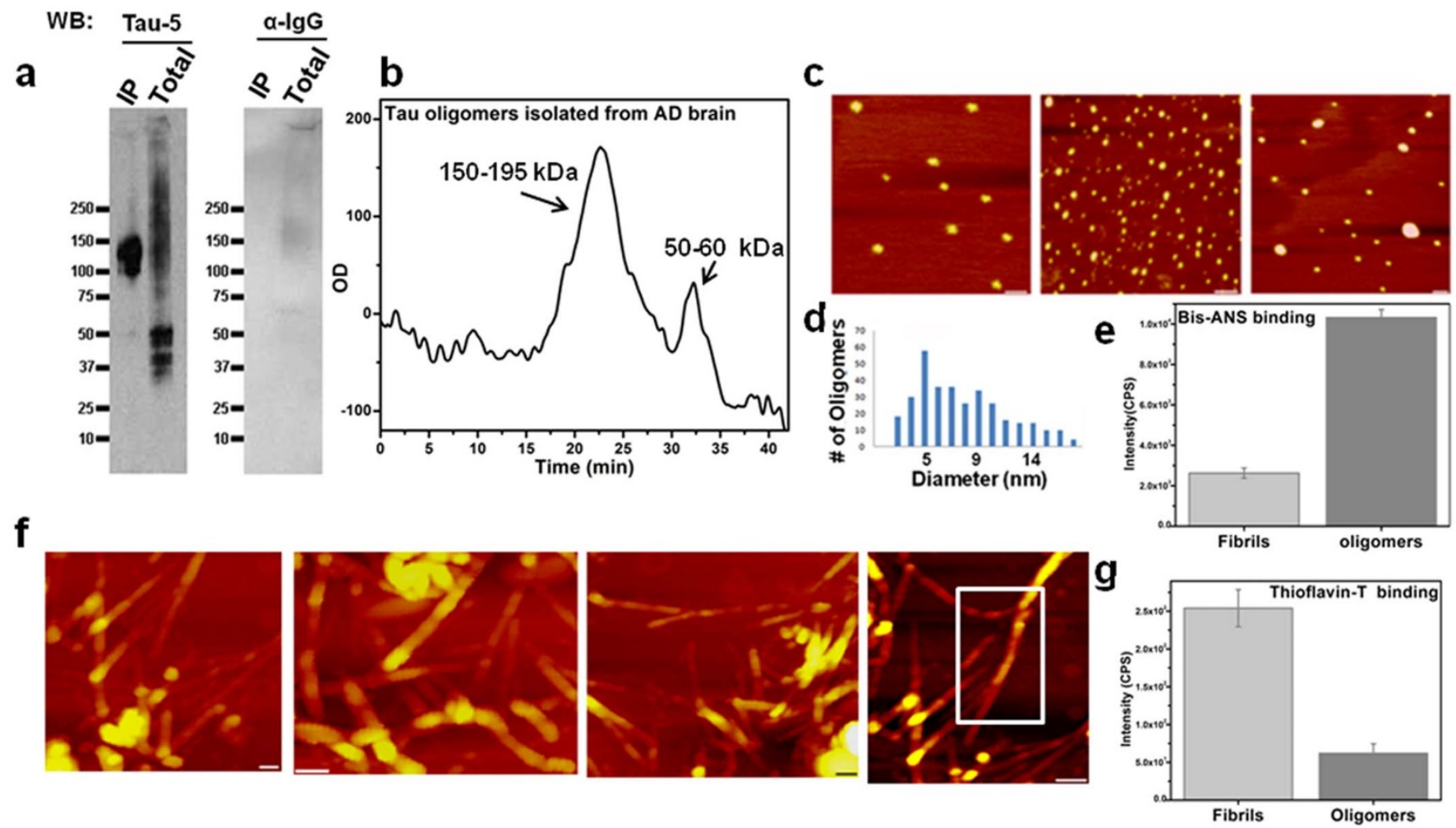

Figure 1 Isolation and characterization of tau oligomers from AD brains. Tau oligomers were isolated by immunoprecipitation (IP) using anti-tau oligomer antibody T22 from the PBS soluble fractions of AD brain homogenates. (a) Western blot using Tau 5 showed that the isolated material (IP) was indeed tau oligomers, without monomer contamination which was abundant in the (Total) PBS soluble fractions from AD brains. The same blot was performed using a control anti-IgG antibody, which failed to detect any non-specific bands. (b) FPLC chromatogram of brain-derived tau oligomers; the main peak is $\sim 150-195 \mathrm{kDa}$ (tau dimer/trimer). The same protocol was used for further purification of oligomers isolated by IP. (c) AFM analysis showing brain-derived tau oligomers isolated from 3 different AD brains. (d) Size distribution histogram of AD brain-derived tau oligomers shows that the majority of these oligomers have a diameter of 4-8 $\mathrm{nm}$. (e) Brain-derived oligomers have higher affinity for Bis-ANS than brain-derived fibrils, which implies that oligomers display more hydrophobic patches. (f) AFM images of brain-derived PHF isolated from the sarcosyl insoluble fractions of AD brain homogenates, the representative images show that they are classical PHF with many twists (white rectangle). (g) Brain-derived PHF show strong ThT binding affinity as compared to brain-derived oligomers, which confirms fibrillar nature of PHF. 
seed the aggregation of full length recombinant human tau in vitro ${ }^{23}$. Here, we demonstrate that tau oligomers isolated from human $\mathrm{AD}$ brain induce the aggregation of monomeric recombinant tau in vitro, and that this aggregation is more rapid and robust than that observed using preformed recombinant tau oligomers, as quantified by direct ELISA using T22 anti-tau oligomer antibody (Fig. 2a). Tau oligomers prepared using brain-derived oligomer seeds $(24 \mathrm{hrs})$ were further characterized by size-exclusion chromatography (Fig. 3b), it is clear that at this time point the solution contains homogeneous population of tau oligomers (dimer/trimer), this was confirmed by AFM (inset). Biophysical characterization by circular dichroism (CD) spectroscopy (Fig. 2c), revealed that tau oligomers are $\beta$ sheet rich, this conformation is associated with amyloid ${ }^{17}$ and tau oligomers $^{23}$ toxicity. Moreover, oligomers formed by seeding recombinant tau with brain-derived oligomer seeds are highly toxic, and this toxicity can be rescued by pre-incubation of the oligomers with the anti-tau oligomer antibody T22 ${ }^{18}$ (Fig. $2 \mathrm{~d}$ ). The tau oligomers prepared by seeding with brain-derived tau oligomers
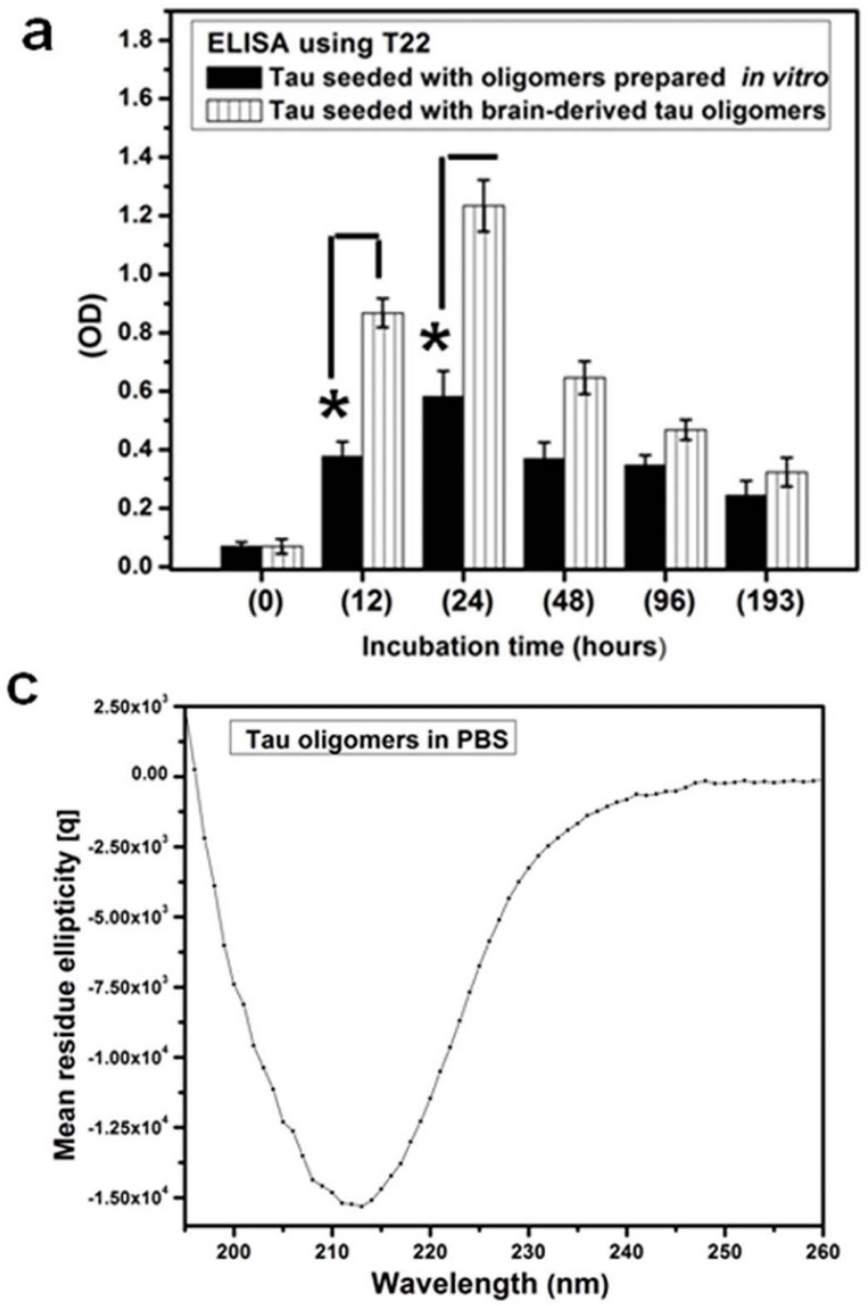

were toxic at sub-micromolar concentrations, yielding oligomers that were 5 times more toxic on a mole for mole basis as compared to oligomers prepared by seeding with recombinant tau oligomers. Collectively, ELISA, SEC, AFM and CD analyses (Fig. 2a-c) show rapid formation of tau oligomers, with no detectable monomer contamination or fibrils formation $24 \mathrm{hrs}$ after seeding initiation. This seeding reaction suggests that brain-derived tau oligomers seed and propagate by an oligomer-nucleated conformational conversion mechanism, similar to what has been proposed for Sup35 prions and recently for $A \beta$ oligomers ${ }^{37,39}$. Unlike the seeding mechanism of tau fibrils (template-assisted growth) ${ }^{35,40}$, this mechanism excludes the addition of tau monomers to the nucleus/template, which provides a template for all monomers to assemble into oligomers before fibril formation ${ }^{23,37,39}$.

Tau oligomers acute effects on hippocampal long term potentiation (LTP). Next we studied the effect of brain-derived tau oligomers on synaptic plasticity in the CA1 region of the hippocampus.
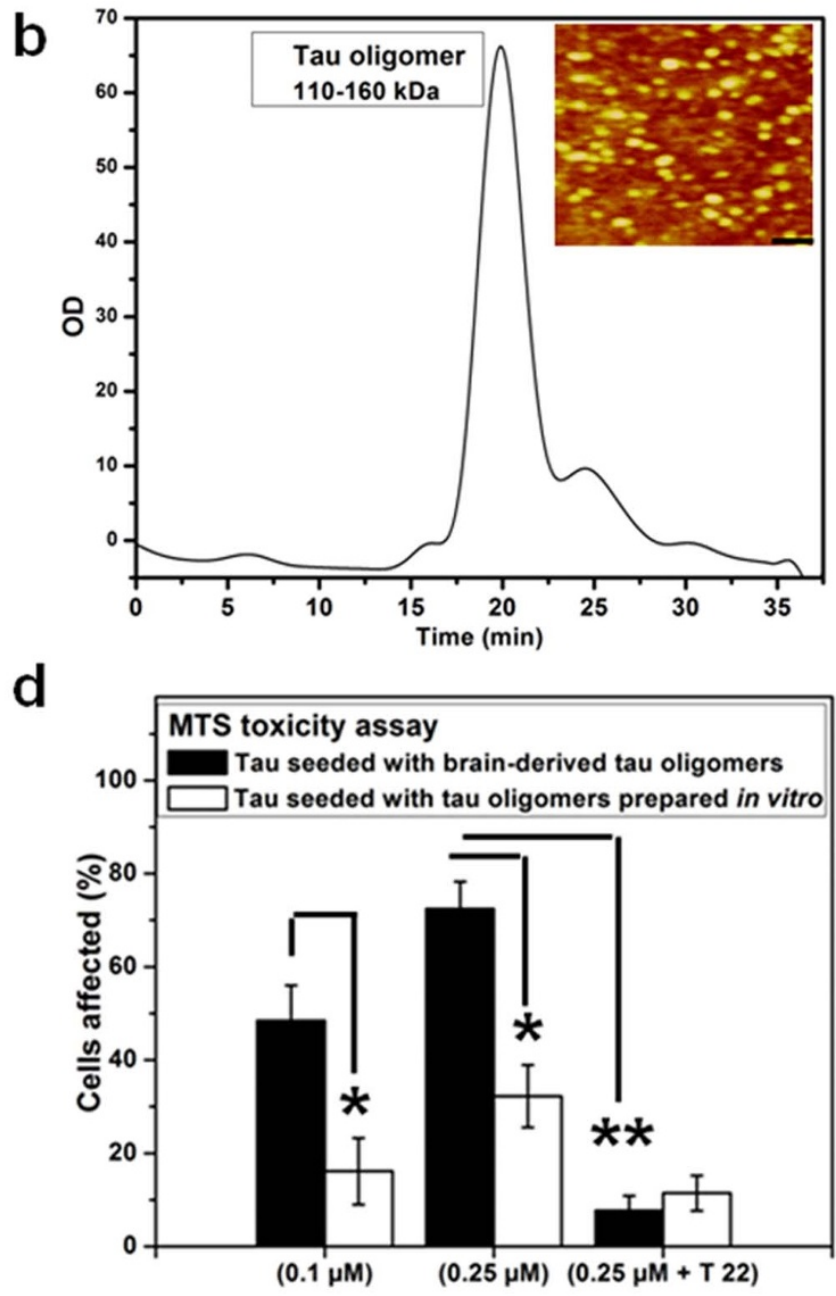

Figure 2 Brain-derived tau oligomers propagate in vitro. (a) Monomeric recombinant full-length tau was seeded (70/1 wt/wt) by either brain-derived oligomers (striped bars) or tau oligomers prepared in vitro (black). AD brain-derived tau oligomers induced aggregation of recombinant tau monomer as quantified by ELISA using T22; brain derived tau oligomers are more potent seeds than the oligomers prepared in vitro, after 24 hrs incubation monomeric tau fully assembled into oligomers. (b) Tau oligomers prepared using brain-derived oligomer seeds (24 hrs) were characterized by SEC; it is clear that at this time point the solution contains homogeneous population of tau oligomers (dimer/trimer). This was confirmed by AFM (inset). Scale bar, $10 \mathrm{~nm}$. (c) Circular dichroism (CD) spectroscopy of tau oligomers formed after 24 hrs incubation $(0.2 \mathrm{mg} / \mathrm{ml}$ in PBS) demonstrates that tau oligomers are $\beta$-sheet rich with minimum ellipticity $(\sim 213 \mathrm{~nm})$. (d) Tau oligomers prepared using brain-derived oligomer seeds were more toxic to cells (at a much lower concentration) than oligomers prepared using seeds prepared from recombinant tau in vitro. Preincubation of tau oligomers with T22 $(1: 4(\mathrm{~mol} / \mathrm{mol})$ for $1 \mathrm{hr})$ eliminated their toxicity as assessed by dye reduction assay using neuroblastoma cells. (** $P<0.001$; * $P<0.01$, two-way ANOVA). 

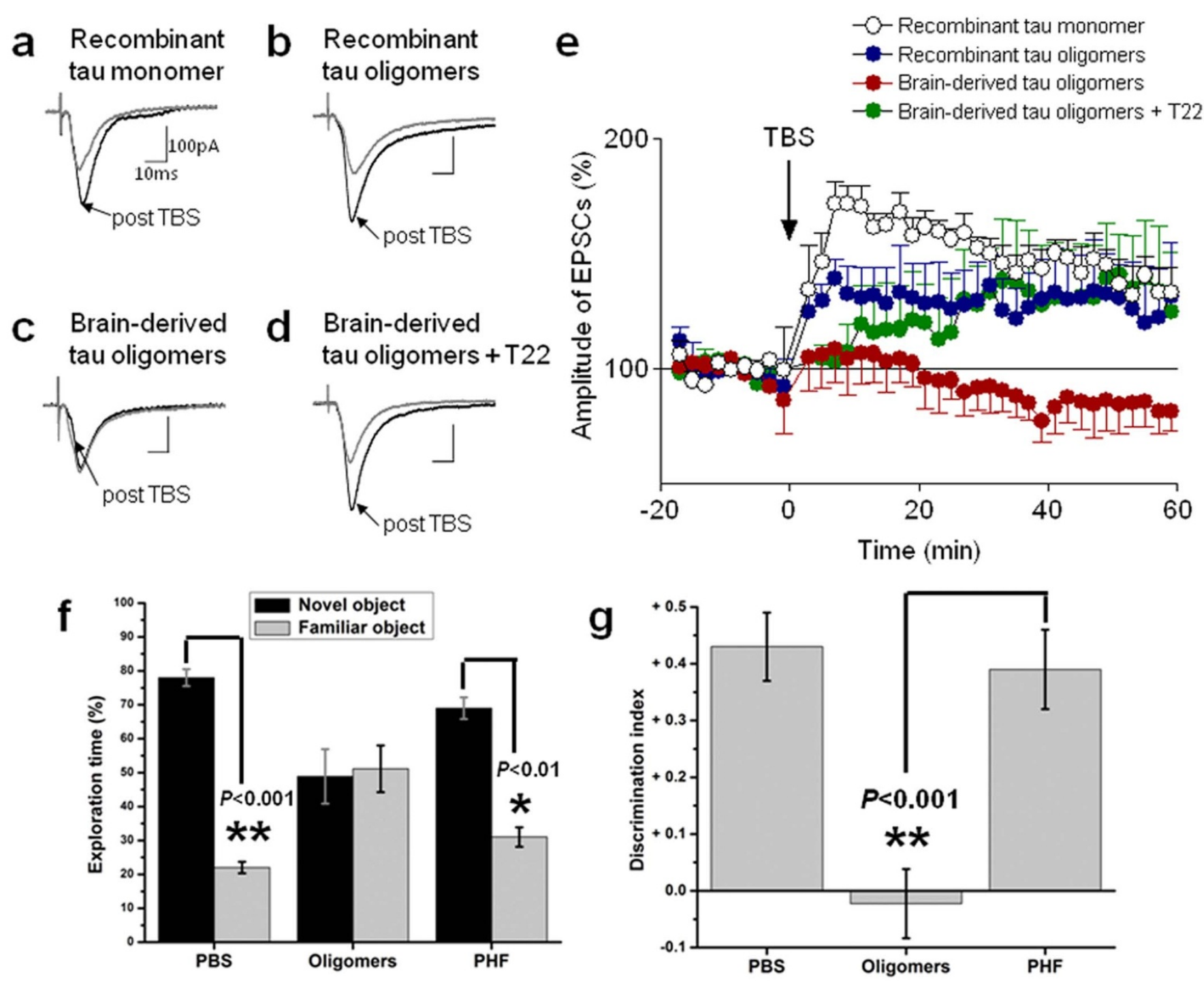

Figure 3 Brain-derived tau oligomers are potent inhibitors of long term potentiation (LTP) in hippocampal brain slices, and repress the recognition memory in vivo. Original recordings of excitatory postsynaptic currents (EPSCs) evoked in individual CA1 pyramidal cells before and after theta burst stimulation (TBS). The individual neurons were recorded in (a) a control hippocampal slice pretreated with recombinant tau monomer, (b) in a hippocampal slice pretreated with recombinant tau oligomers, (c) in a hippocampal slice pretreated with brain-derived tau oligomers, and (d) in a hippocampal slice pretreated with brain-derived tau oligomers preincubated with tau oligomer-specific antibody (T22). Each trace is the average of $8-10$ EPSCs. Scale bars, 100 pA, 10 ms. (e) Time course of LTP averaged across the sample of neurons recorded in ( $a, n=6$ neurons), (b, n=5 neurons), (c, $\mathrm{n}=7$ neurons), and ( $\mathrm{d}, \mathrm{n}=6$ neurons). Each symbol shows the mean \pm SEM of 10 EPSCs at every 30 s. Brain-derived tau oligomers, but not brainderived PHF, impair object recognition memory in vivo. (f) Brain-derived tau oligomers and PHF isolated and characterized as shown in figure 1 were injected into the hippocampus bilaterally $(0.6 \mu \mathrm{g}$ each). Mice were tested 3 days post injection. Mice injected with PBS, and PHF spent significantly more time investigating the novel object, whereas mice injected with brain-derived tau oligomers showed memory impairment, as evidenced by their inability to recognize the novel object and hence spending equal time investigating both objects. (g) Histograms show the discrimination index corresponding to the data in (f).

Hippocampal long term potentiation (LTP) was measured using whole cell patch-clamp recordings of excitatory postsynaptic currents (EPSCs) evoked at the CA3-CA1 synapse that is formed by axons in the Schaffer collateral-commissural pathway (Fig. 3), as we described previously ${ }^{41}$. A robust form of LTP was induced in untreated brain slices using a theta burst stimulation (TBS) protocol ${ }^{42}$. No effects on LTP were observed in hippocampal slices pretreated with recombinant tau monomer at $100 \mathrm{nM}$ final concentration (Fig. 3a). In contrast, LTP was decreased in slices pretreated with recombinant tau oligomers at $50 \mathrm{nM}$ (Fig. 3b). In slices pretreated with brain-derived tau oligomers (50 nM final concentration; Fig. 3c), the brain-derived oligomers were significantly more potent than oligomers prepared from recombinant tau in vitro $(p<$
0.0001, two-way ANOVA). Importantly, preincubation of brainderived oligomers with an excess amount of the tau oligomerspecific antibody $\mathrm{T} 22^{18}$ restore a delayed onset of LTP in treated hippocampal slices $(p<0.0001$, two-way ANOVA). These data suggest that brain-derived tau dramatically impairs synaptic function and that this toxic effect can be rescued by the physical binding of our antibody.

Pathological effects of tau oligomers in vivo. We investigated the pathological relevance of brain-derived tau oligomers in vivo and their ability to seed the aggregation and propagation of endogenous tau in wild type mice by injecting either pure tau oligomers or PHF tau isolated from AD human brains into the hippocampus of 
3 months old wild type C57BL/6 mice. Both tau species were fully characterized as described in Figure 1. Each of C57BL/6 mice (n-12) received a single injection into each hemisphere with brain-derived oligomeric tau. Whereas a second group of $(n=12)$ was injected with PHF $(0.6 \mu \mathrm{g} /$ hemisphere $)$. A third group $(n=12)$ was injected with PBS to control for any unexpected effects from the injection procedure. Finally, a fourth group consisting of 3 months old tau knockout mice $(n=8)^{43}$ were injected as controls to ascertain the role of endogenous tau in the acute toxicity of tau oligomers.

To evaluate the effects of brain-derived tau oligomers and PHF on memory, we performed the novel object recognition behavior test 3 days post injection ${ }^{22,44}$. Briefly, animals were trained in an arena containing two objects that they could explore freely (familiarization phase), 6 hours later, we exposed them to one familiar and one new object (test phase). Mice injected with brain-derived tau oligomers were unable to distinguish the new object, with no significant difference in the percentage of time spent investigating both objects (Fig. 3f), and displayed a discrimination index significantly lower than that of the PHF injected group (Fig. 3g). Neither PBS nor any of the PHF treated C57BL/6 animals showed memory deficits in this task (Fig. 3f and g). These data indicate that tau oligomers isolated from AD human brain acutely disrupt anterograde memory storage $^{22,44,45}$. No memory deficits were found in the tau knockout mice injected with brain-derived oligomers (Supplementary Fig.S1a and b) demonstrating that endogenous tau plays a critical role in mediating tau oligomers toxicity in vivo.

To determine if behavioral deficits persist over time in the mice injected with brain-derived tau oligomers, a novel object recognition task was performed again 11 months post injection. No statistically significant behavioral deficit was observed in any of the groups, demonstrating that mice injected with tau oligomers were able to fairly recover from the acute deficit produced by tau oligomers (Supplementary Fig. S1c and d). Analysis of mouse brains 11 months post injection unexpectedly revealed that brain-derived oligomers induced widespread filamentous/ amyloidogenic tau pathology in C57BL/6 mice, as clearly indicated by the appearance of thioflavin $\mathrm{S}$ (ThS) staining (Fig. 4a-b and supplementary Fig. S2a-b) and Gallyas silver (Fig. 4c-d, Supplementary Fig S2c-d-f and supplementary Fig. S3c $)^{46,47}$. In addition to the positive ThS and silver staining, the administration of brain-derived oligomers resulted in the appearance of AT8 immunoreactivity (Fig. 4e-f, supplementary Fig S2e-f and Fig. S3f), which is indicative of hyperphosphorylated tau filaments ${ }^{48}$. Positive staining for tau deposits did not remain confined to the injected area (hippocampus), but spread to neighboring brain regions such as the cortex (Fig. 4), corpus callosum and hypothalamus (Supplementary Fig. S2). Immunoreactivity for the human tau-specific monoclonal antibody HT7 (Fig. 4g-j) was present only at the site of the injection (Fig. $3 \mathrm{~g}$ ), thus demonstrating that tau deposits in the wild type mice injected with human brain-derived tau oligomers (Fig. 4, supplementary Fig. S2 and Fig S3) are of murine origin. To confirm these observations and exclude the possibility that the aggregates observed were unrelated to the injected material, additional groups of mice $(n=6)$ were injected and sacrificed 3 days post injection. Tau hyperphosphorylated deposits were not detected in any of the mice by immunohistochemical analysis, confirming that tau deposits observed are derived from murine tau (Supplementary Fig. S4a-d). Mice injected with PHF tau showed AT8 immunoreactivity 11 months post injection only at the injection site (Supplementary Fig. S4e-f), suggesting that fibrillar tau is unable to act as a seed to induce tau propagation in a prion-like mechanism.

\section{Discussion}

While it is now evident that tau aggregation can be induced exogenously in vivo using brain extracts containing tau aggregates ${ }^{5,49}$, at this time it is unknown which of the various misfolded species of tau is most efficient in triggering tau aggregation. The data presented here demonstrate that soluble forms of tau known as tau oligomers not only induce toxicity but also act as the key species to induce tau

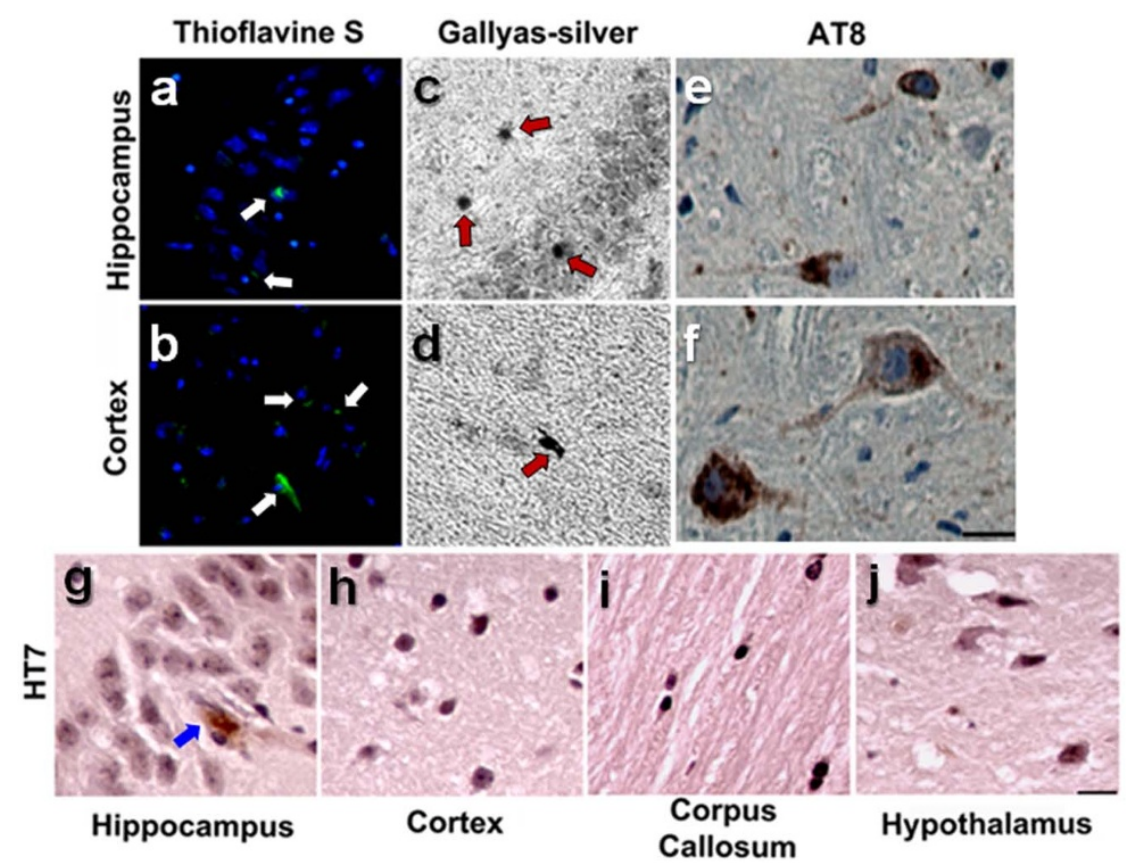

Figure $4 \mid$ Brain-derived tau oligomers are potent seeds inducing the aggregation and propagation of endogenous tau in vivo. Only mice injected with brain-derived tau oligomers displayed significant widespread tau pathology 11 months post injection. Tau pathology was detected using well established methods, including ThS and Gallyas silver, as well as immunohistochemistry using AT8 and HT7 antibodies. (a-f) NFT in the hippocampal CA1 region and frontal cortex. Positive staining with Gallyas silver, ThS, and AT8 (specific for Ser202/Thr205 phosphorylated tau) established the presence of hyperphosphorylated NFT deposits and the spreading of the pathology to areas far from the injection site (hippocampus). (g-j) HT7 staining (specific for human tau) was confined to the injection site (g), demonstrating that tau deposits in neighboring areas are comprised of endogenous murine tau and not derived from the original inocula. Scale bars $12 \mu \mathrm{m}$. 
pathology propagation in wild type mice via a mechanism reminiscent of that used by prions, rather than simply acting to accelerate pathology induced by transgenes in mice, as has been described previously ${ }^{3,5}$. It is important to keep in mind that this observation of tauopathy propagation in wild type mice is more relevant to sporadic $\mathrm{AD}$, since tau mutations are not associated with $\mathrm{AD}$ but rather with frontotemporal lobar degeneration.

We observed previously that tau oligomers prepared in vitro from full length recombinant human tau effectively induce behavioral deficits in C57BL/6 mice after intracerebral administration ${ }^{22}$. Nevertheless, these oligomers did not induce endogenous tau propagation after 11 months post injection (Lasagna-Reeves and Kayed, unpublished observations), despite the fact that both have identical amino acid sequences. This result suggests that tau oligomers in vivo either assume a multidimensional very stable conformation as compared to that of tau oligomers prepared in vitro, or that posttranslational modifications and/or cofactors in the brain are essential for tau oligomers to act as seeds to promote propagation. The ability of mice to recover from acute behavioral deficits after the administration of human brain-derived tau oligomers could be explained by the recent observation of E.-M. Mandelkow and coworkers using a conditional mouse model of tauopathy that toxicity is linked to expression of proaggregant tau rather than aggregation per $\mathrm{se}^{11}$. This finding suggests that tau oligomers injected intracerebrally mimic the expression of pro-aggregant toxic tau and that further administration of this tau species is necessary to maintain behavioral impairment in the mice. Moreover, this recovery may be indicative of the ability of healthy cells in young mice to handle the first wave of tau aggregation by forming non-toxic large fibrillar deposits, thus it is likely that cell death and neurodegeneration will arise at much later stage. Consistent with this interpretation, the fact that neurons can live for decades with neurofibrillary tangles (NFT) ${ }^{50}$, and Braak and Del Tredici serendipitously identified AT8 and Gallyas positive tau structures in brains from children and young adults ${ }^{51,52}$.

In this study the term "prion-like" depicts the self-propagation of amyloidogenic proteins across cells and tissues, and does not imply that these aggregated proteins are infectious ${ }^{53}$. The data presented here suggest that the behavior of tau aggregates bears resemblance to that of prions, in which non-fibrillar small particles have been identified as necessary for propagation ${ }^{54}$. Indeed, as is the case for prions, it is possible that a range of tau species constitute the templating and toxic entities and that these populations may overlap ${ }^{55,56}$. Hence, these results may impact a variety of fields as the concept of prionlike induction and spreading of pathogenic proteins recently has been proposed for many neurodegenerative diseases ${ }^{57,58}$. Drugs that target amyloid and tau oligomers may be valuable for the treatment of a variety of neurodegenerative disease and preventing spread of pathology.

1. Brundin, P., Melki, R. \& Kopito, R. Prion-like transmission of protein aggregates in neurodegenerative diseases. Nat Rev Mol Cell Biol 11, 301-307 (2010).

2. Frost, B. \& Diamond, M. I. Prion-like mechanisms in neurodegenerative diseases. Nat Rev Neurosci 11, 155-159 (2010).

3. Meyer-Luehmann, M. et al. Exogenous induction of cerebral betaamyloidogenesis is governed by agent and host. Science 313, 1781-1784 (2006).

4. Eisele, Y.S. et al. Peripherally applied Abeta-containing inoculates induce cerebral beta-amyloidosis. Science 330, 980-982 (2010).

5. Clavaguera, F. et al. Transmission and spreading of tauopathy in transgenic mouse brain. Nat Cell Biol 11, 909-913 (2009).

6. Liu, L. et al. Trans-synaptic spread of tau pathology in vivo. PLoS One 7, e31302 (2012).

7. de Calignon, A. et al. Propagation of tau pathology in a model of early Alzheimer's disease. Neuron 73, 685-697 (2012).

8. Kosik, K. S., Orecchio, L. D., Bakalis, S. \& Neve, R. L. Developmentally regulated expression of specific tau sequences. Neuron 2, 1389-1397 (1989).

9. Giasson, B. I. et al. Initiation and synergistic fibrillization of tau and alphasynuclein. Science 300, 636-640 (2003).

10. Kurt, M. A., Davies, D. C., Kidd, M., Duff, K. \& Howlett, D. R. Hyperphosphorylated tau and paired helical filament-like structures in the brains of mice carrying mutant amyloid precursor protein and mutant presenilin-1 transgenes. Neurobiol Dis 14, 89-97 (2003).

11. Sydow, A. et al. Tau-induced defects in synaptic plasticity, learning, and memory are reversible in transgenic mice after switching off the toxic Tau mutant. J Neurosci 31, 2511-2525 (2011).

12. Roberson, E. D. et al. Reducing endogenous tau ameliorates amyloid beta-induced deficits in an Alzheimer's disease mouse model. Science 316, 750-754 (2007).

13. Vossel, K. A. et al. Tau reduction prevents Abeta-induced defects in axonal transport. Science 330, 198 (2010).

14. Nussbaum, J. M. et al. Prion-like behaviour and tau-dependent cytotoxicity of pyroglutamylated amyloid-beta. Nature 485, 651-655 (2012).

15. Haass, C. \& Selkoe, D. J. Soluble protein oligomers in neurodegeneration: lessons from the Alzheimer's amyloid beta-peptide. Nat Rev Mol Cell Biol 8, 101-112 (2007).

16. Caughey, B., Baron, G. S., Chesebro, B. \& Jeffrey, M. Getting a grip on prions: oligomers, amyloids, and pathological membrane interactions. Annu Rev Biochem 78, 177-204 (2009).

17. Kayed, R. et al. Common structure of soluble amyloid oligomers implies common mechanism of pathogenesis. Science 300, 486-489 (2003).

18. Lasagna-Reeves, C. A. et al. Identification of oligomers at early stages of tau aggregation in Alzheimer's disease. FASEB J 26, 1946-1959 (2012).

19. Patterson, K. R. et al. Characterization of prefibrillar Tau oligomers in vitro and in Alzheimer disease. J Biol Chem 286, 23063-23076 (2011).

20. Langer, F. et al. Soluble Abeta seeds are potent inducers of cerebral beta-amyloid deposition. J Neurosci 31, 14488-14495 (2011).

21. Lasagna-Reeves, C. A. et al. Identification of oligomers at early stages of tau aggregation in Alzheimer's disease. FASEB J 26, 1946-1959 (2012).

22. Lasagna-Reeves, C. A. et al. Tau oligomers impair memory and induce synaptic and mitochondrial dysfunction in wild-type mice. Mol Neurodegener 6, 39 (2011).

23. Lasagna-Reeves, C. A., Castillo-Carranza, D. L., Guerrero-Muoz, M. J., Jackson, G. R. \& Kayed, R. Preparation and characterization of neurotoxic tau oligomers. Biochemistry 49, 10039-10041 (2010).

24. Prusiner, S. B. Novel proteinaceous infectious particles cause scrapie. Science 216, 136-144 (1982).

25. Prusiner, S. B. et al. Evidence for hydrophobic domains on the surface of the scrapie agent. Trans Am Neurol Assoc 103, 62-64 (1978).

26. Rostagno, A. \& Ghiso, J. Isolation and biochemical characterization of amyloid plaques and paired helical filaments. Curr Protoc Cell Biol Chapter 3, 1-33 (2009).

27. Guo, J. L. \& Lee, V. M. Seeding of normal Tau by pathological Tau conformers drives pathogenesis of Alzheimer-like tangles. J Biol Chem 286, 15317-15331 (2011).

28. Kfoury, N., Holmes, B. B., Jiang, H., Holtzman, D. M. \& Diamond, M. I. Trans-cellular propagation of Tau aggregation by fibrillar species. J Biol Chem 287, 1440-1451 (2012).

29. Berger, Z. et al. Accumulation of pathological tau species and memory loss in a conditional model of tauopathy. J Neurosci 27, 3650-3662 (2007).

30. Spires, T. L. et al. Region-specific dissociation of neuronal loss and neurofibrillary pathology in a mouse model of tauopathy. Am J Pathol 168, 1598-1607 (2006).

31. Jarrett, J. T., Berger, E. P. \& Lansbury, P. T. Jr. The carboxy terminus of the beta amyloid protein is critical for the seeding of amyloid formation: implications for the pathogenesis of Alzheimer's disease. Biochemistry 32, 4693-4697 (1993).

32. Kelly, J. W. Mechanisms of amyloidogenesis. Nat Struct Biol 7, 824-826 (2000).

33. O'Nuallain, B., Williams, A. D., Westermark, P. \& Wetzel, R. Seeding specificity in amyloid growth induced by heterologous fibrils. J Biol Chem 279, 17490-17499 (2004).

34. Dinkel, P. D., Siddiqua, A., Huynh, H., Shah, M. \& Margittai, M. Variations in filament conformation dictate seeding barrier between three- and four-repeat tau. Biochemistry 50, 4330-4336 (2011).

35. Margittai, M. \& Langen, R. Template-assisted filament growth by parallel stacking of tau. Proc Natl Acad Sci U S A 101, 10278-10283 (2004).

36. Siddiqua, A. \& Margittai, M. Three- and four-repeat Tau coassemble into heterogeneous filaments: an implication for Alzheimer disease. J Biol Chem 285, 37920-37926 (2010).

37. Lee, J., Culyba, E. K., Powers, E. T. \& Kelly, J. W. Amyloid-beta forms fibrils by nucleated conformational conversion of oligomers. Nat Chem Biol 7, 602-609 (2011).

38. Kayed, R. et al. Conformation dependent monoclonal antibodies distinguish different replicating strains or conformers of prefibrillar Abeta oligomers. $\mathrm{Mol}$ Neurodegener 5, 57 (2010).

39. Serio, T. R. et al. Nucleated conformational conversion and the replication of conformational information by a prion determinant. Science 289, 1317-1321 (2000).

40. Congdon, E. E. et al. Nucleation-dependent tau filament formation: the importance of dimerization and an estimation of elementary rate constants. J Biol Chem 283, 13806-13816 (2008)

41. Dineley, K. T. et al. Amyloid-beta oligomers impair fear conditioned memory in a calcineurin-dependent fashion in mice. J Neurosci Res 88, 2923-2932 (2012).

42. Cao, G. \& Harris, K. M. Developmental regulation of the late phase of long-term potentiation (L-LTP) and metaplasticity in hippocampal area CA1 of the rat. J Neurophysiol 107, 902-912 (2012).

43. Tucker, K. L., Meyer, M. \& Barde, Y. A. Neurotrophins are required for nerve growth during development. Nat Neurosci 4, 29-37 (2001). 
44. Balducci, C. et al. Synthetic amyloid-beta oligomers impair long-term memory independently of cellular prion protein. Proc Natl Acad Sci U S A 107, 2295-2300 (2010).

45. Greene, J. D., Baddeley, A. D. \& Hodges, J. R. Analysis of the episodic memory deficit in early Alzheimer's disease: evidence from the doors and people test. Neuropsychologia 34, 537-551 (1996).

46. Gallyas, F., Guldner, F. H., Zoltay, G. \& Wolff, J. R. Golgi-like demonstration of "dark" neurons with an argyrophil III method for experimental neuropathology. Acta Neuropathol 79, 620-628 (1990).

47. LeVine, H. 3rd. Quantification of beta-sheet amyloid fibril structures with thioflavin T. Methods Enzymol 309, 274-284 (1999).

48. Augustinack, J. C., Schneider, A., Mandelkow, E. M. \& Hyman, B. T. Specific tau phosphorylation sites correlate with severity of neuronal cytopathology in Alzheimer's disease. Acta Neuropathol 103, 26-35 (2002).

49. Prusiner, S. B. Cell biology. A unifying role for prions in neurodegenerative diseases. Science 336, 1511-1513 (2012).

50. Morsch, R., Simon, W. \& Coleman, P. D. Neurons may live for decades with neurofibrillary tangles. J Neuropathol Exp Neurol 58, 188-197 (1999).

51. Braak, H. \& Del Tredici, K. The pathological process underlying Alzheimer's disease in individuals under thirty. Acta Neuropathol 121, 171-181 (2011)

52. Duyckaerts, C. Tau pathology in children and young adults: can you still be unconditionally baptist? Acta Neuropathol 121, 145-147 (2011).

53. Hardy, J. \& Revesz, T. The spread of neurodegenerative disease. NEngl J Med 366 2126-2128 (2012).

54. Silveira, J. R. et al. The most infectious prion protein particles. Nature 437, 257-261 (2005)

55. Collinge, J. \& Clarke, A. R. A general model of prion strains and their pathogenicity. Science 318, 930-936 (2007).

56. Li, J., Browning, S., Mahal, S. P., Oelschlegel, A. M. \& Weissmann, C. Darwinian evolution of prions in cell culture. Science 327, 869-872 (2010).

57. Polymenidou, M. \& Cleveland, D. W. The seeds of neurodegeneration: prion-like spreading in ALS. Cell 147, 498-508 (2011).
58. Munch, C. \& Bertolotti, A. Propagation of the prion phenomenon: beyond the seeding principle. J Mol Biol 421, 491-498 (2012).

\section{Acknowledgments}

We thank Dr. Bridget Hawkins for useful suggestions. We are grateful to Shashirekha Krishnamurthy and Malika Farhed for excellent technical assistance, as well as to Drs. Adriana Paulucci, Mathieu Bakhoum, and Yogesh Wairkar for their help with microscopy and image analysis. This work was supported by: The Cullen Family Trust for Health Care, the Alzheimer's Drug Discovery Foundation (ADDF), the Michael J. Fox Foundation, and the Mitchell Center for Neurodegenerative Diseases.

\section{Author contributions}

R.K. conceived the project and developed the tau oligomer antibodies. C.A.L., D. L.C., G.R.J. and R.K. planned the experiments and discussed the results. C.A.L., U.S., M.J.M. and R.K. isolated and characterized tau species from $\mathrm{AD}$ and performed the in vitro experiments. T.K. and V.N. performed the LTP experiments and analysis. C.A.L. and D.L.C. performed the injections, behavioral analysis, and the IHC. C.A.L., D.L.C., V.N., G.R.J. and R.K prepared the figures. C.A.L., G.R.J. and R.K. wrote the manuscript.

\section{Additional information}

Supplementary information accompanies this paper at http://www.nature.com/ scientificreports

Competing financial interests: R. K. has patent applications on the compositions and methods related to tau oligomers and antibodies.

License: This work is licensed under a Creative Commons

Attribution-NonCommercial-ShareAlike 3.0 Unported License. To view a copy of this

license, visit http://creativecommons.org/licenses/by-nc-sa/3.0/

How to cite this article: Lasagna-Reeves, C.A. et al. Alzheimer brain-derived tau oligomers propagate pathology from endogenous tau. Sci. Rep. 2, 700; DOI:10.1038/srep00700 (2012). 